\title{
Ground-based visible and near-IR observations of Comet 9P/Tempel 1 during the Deep Impact encounter
}

\author{
Matthew M. Knight a, ,,1, Kevin J. Walsh ${ }^{\text {a }}$, Michael F. A'Hearn ${ }^{\text {a }}$, Robert A. Swaters ${ }^{\text {a, },}$, \\ B. Ashley Zauderer ${ }^{\mathrm{a}}$, Nalin H. Samarasinha ${ }^{\mathrm{b}, \mathrm{c}, 2}$, Roberto Vázquez ${ }^{\mathrm{d}}$, Harold Reitsema ${ }^{\mathrm{e}}$ \\ ${ }^{a}$ Department of Astronomy, University of Maryland, College Park, MD 20742-2421, USA \\ b National Optical Astronomy Observatory, 950 N. Cherry Ave., Tucson, AZ 85719, USA \\ ${ }^{c}$ Planetary Science Institute, 1700 E. Fort Lowell, Suite 106, Tucson, AZ 85719, USA \\ d Instituto de Astronomía, Universidad Nacional Autónoma de México, Km 103 Carretera Tijuana-Ensenada, 22860 Ensenada, B.C., Mexico \\ ${ }^{\mathrm{e}}$ Ball Aerospace, 1600 Commerce St., Boulder, CO 80306-1062, USA
}

Received 15 April 2006; revised 18 September 2006

\begin{abstract}
We present the results of our visible and near-IR observations of Comet 9P/Tempel 1 during the Deep Impact encounter. The comet was observed before, during, and after impact from Kitt Peak National Observatory (J, H, K) and Observatorio Astronómico Nacional-San Pedro Mártir, Mexico (B, V, R, I). High time-resolution images in R, J, H, and $\mathrm{K}$ the night of impact with a 3." 5 radius aperture revealed a rapid brightening which had multiple slopes and lasted for approximately $25 \mathrm{~min}$ before leveling off. The brightness decreased on subsequent nights and returned to near pre-impact levels by July 8 UT. The R-J, R-H, R-K, J-H, J-K, and H-K colors became bluer the night of impact. The R-J, R-H, and R-K colors remained blue on the night after impact while the $\mathrm{J}-\mathrm{H}, \mathrm{J}-\mathrm{K}$, and $\mathrm{H}-\mathrm{K}$ colors returned to baseline levels. The observed color changes suggest the bluening was due to an increase in small grains relative to the ambient coma, an increase in ice relative to refractory dust in the coma, or a combination of the two. The ejecta were initially directed towards the southwest but had been driven southeast by solar radiation pressure by the second night after impact. The mean projected ejecta velocity was estimated at $0.20-0.23 \mathrm{~km} \mathrm{~s}^{-1}$ over the first $24 \mathrm{~h}$ after impact.
\end{abstract}

(C) 2006 Elsevier Inc. All rights reserved.

Keywords: Comet Tempel-1

\section{Introduction}

Discovered in 1867 by the German astronomer Ernst Tempel, Comet 9P/Tempel 1 was observed on its subsequent perihelion passages in 1873 and 1879. It underwent a close approach

\footnotetext{
This article originally appeared in Icarus Vol. 187/1, March 2007, pp. 199207. Those citing this article should use the original publication details.

* Corresponding author. Fax: +1 3013149067.

E-mail address: mmk8a@astro.umd.edu (M.M. Knight).

1 Visiting Astronomer, Kitt Peak National Observatory, National Optical Astronomy Observatory, which is operated by the Association of Universities for Research in Astronomy, Inc. (AURA) under cooperative agreement with the National Science Foundation.

2 Kitt Peak National Observatory, National Optical Astronomy Observatory, which is operated by the Association of Universities for Research in Astronomy, Inc. (AURA) under cooperative agreement with the National Science Foundation.
}

to Jupiter in 1881, increasing its perihelion distance to $2.1 \mathrm{AU}$, and causing it to elude further observation until 1967. Additional close approaches to Jupiter in 1941 and 1953 reduced the perihelion distance to $1.5 \mathrm{AU}$, resulting in a relatively stable orbit which has yielded observations at all seven of the comet's returns since 1967 (Yeomans et al., 2005), with alternate orbits having favorable geometries for Earth-based observatories. Tempel 1 was selected as the target body for the Deep Impact mission due to its favorable dynamics, large nuclear size, and observability from the Earth at the time of the Deep Impact encounter (A'Hearn et al., 2005a).

NASA's Deep Impact spacecraft was launched on January 12, 2005, encountering Comet 9P/Tempel 1 on July 4, 2005 UT at a heliocentric distance of $1.506 \mathrm{AU}$ and a geocentric distance of 0.894 AU. The impact was scheduled to be observable from Hawaii, the western United States, and by space telescopes such as Hubble and Spitzer (A'Hearn et al., 2005a). 
The scientific results were augmented by observations made by observers around the world in wavelengths and timescales unavailable to the spacecraft. As part of the extensive groundbased observing campaign in support of the mission (Meech et al., 2005a, 2005b), we observed the comet in the days prior to, during, and after impact from Kitt Peak National Observatory $(\mathrm{J}, \mathrm{H}, \mathrm{K})$ and Observatorio Astronómico Nacional at the Sierra de San Pedro Mártir, B.C. ${ }^{3}$ (B, V, R, I). Kitt Peak was selected for its Simultaneous Quad Infrared Imaging Device (SQIID) which images simultaneously in $\mathrm{J}, \mathrm{H}, \mathrm{K}$, and L. This allowed the comet's $\mathrm{J}-\mathrm{H}, \mathrm{J}-\mathrm{K}$, and $\mathrm{H}-\mathrm{K}$ colors to be closely monitored during the min immediately after impact, providing information about the composition and evolution of the ejecta. San Pedro Mártir was selected to provide high speed photometry at a location relatively distant from the other facilities observing Deep Impact, providing a site of good weather and seeing conditions for viewing the encounter.

The organization of the paper is as follows. In Section 2 we describe our observations and data reduction. In Section 3 we discuss the lightcurve, examining it over the night of impact and for the following week. In Section 4 we discuss the color change seen on the night of impact. In Section 5 we discuss the nightly changes in coma morphology and estimate the ejecta velocity. In Section 6 we interpret our observations, placing them within the framework of the Deep Impact results as a whole. Our results are summarized in Section 7.

\section{Observations and reductions}

\subsection{Near-IR}

We observed Tempel 1 with the Kitt Peak National Observatory $2.1-\mathrm{m}$ telescope for eight nights beginning on July 2 UT (Table 1). Observations were made using the SQIID infrared camera which simultaneously observes in $\mathbf{J}$ (1.131$1.402 \mu \mathrm{m}), \mathrm{H}(1.535-1.809 \mu \mathrm{m}), \mathrm{K}(1.95-2.30 \mu \mathrm{m})$, and narrowband $\mathrm{L}(3.262-3.336 \mu \mathrm{m})$. L band images were unusable due to the high sky background in summer. Each filter illuminates a $512 \times 512 \mathrm{CCD}$ chip, and combined with the pixel scale of $0 .{ }^{\prime \prime} 69$ pixel $^{-1}$, yields a field of view of 5'0 $\times 5$ '. 3 (vignetting results in a rectangular field of view). Due to the changing geometry of the Earth-comet system, the plate scale ranged from 442 to $460 \mathrm{~km} \mathrm{pixel}^{-1}$ during the observations, and was $447 \mathrm{~km}$ pixel $^{-1}$ at impact.

Exposure times ranged from 4 to $8 \mathrm{~s}$ with shorter exposure times necessary at higher airmasses due to the increasing sky background. Including internal co-additions $(\sim 10 \mathrm{~ms}$ overhead per co-addition) and instrumental overhead ( $\sim 40 \mathrm{~s}$ of overhead to read out to disk), images were taken approximately every $90 \mathrm{~s}$ the night of impact and every 2 min the rest of the run.

On the night of impact, the exposure sequence was planned to capture the impact during an exposure. We began taking 6-s exposures with 7 co-additions at 5:52:02 UT (impact was

\footnotetext{
3 The Observatorio Astronómico Nacional at the Sierra de San Pedro Mártir (OAN-SPM) is operated by the Instituto de Astronomía of the Universidad Nacional Autónoma de México.
}

predicted at 5:52:13 UT, and occurred at 5:52:02 UT accounting for the light travel time from the comet (A'Hearn et al., 2005b)), eventually switching to 4-s exposures with 10 coadditions when the sky background became too high. Impact occurred at an airmass of $\sim 2.5$. The comet was observed until it reached the telescope limit at just over 8 airmasses, about 85 min after impact.

The same standard star field was observed each night. It was selected from the Persson et al. (1998) catalog of near-IR standard fields for its proximity to the comet in both RA $(\alpha)$ and Dec $(\delta)$, allowing standard star observations to be interspersed with comet observations. The nightly range of airmasses for the standard star field and the comet are given in Table 1. The PSF in $\mathrm{J}, \mathrm{H}$, and $\mathrm{K}$ typically evolved from $\sim 1$.' 4 at low airmass to $\sim 2$." 2 at high airmass. A circular 3." 5 radius aperture was selected for all photometry to minimize the fraction of flux lost in the wings of the PSF.

Images were processed using the IRAF UPSQIID package by subtracting a bias and a dark frame, dividing by a flat field constructed from standard star fields and off source pointings, correcting for the non-linearity of the chip, ${ }^{4}$ and normalizing by a median background. Nightly atmospheric extinction corrections were calculated using the standard star fields, with additional standard star photometry obtained from 2MASS (Skrutskie et al., 2006). We did not correct for non-linear extinction, and used only images taken below 2 airmasses for photometry, except on the night of impact where this was impossible. Nightly median images were created by normalizing exposure times and taking the median value at each pixel.

\subsection{Visible}

We observed Tempel 1 with the Observatorio Astronómico Nacional-San Pedro Mártir (Mexico) 1.5-m telescope over four nights, beginning on July 2 UT (Table 1). The observations were made in the broad-band filters B, V, R, and I, and were exclusively in R immediately following impact on July 4 UT. The detector was a $1024 \times 1024$ SITe CCD chip with 0. ' 274 pixel $^{-1}$ scale and a $4.7 \times 4.7$ field of view.

Exposures were typically $20 \mathrm{~s}$, cycling through the B, V, R, and I filters. The imaging sequence on the night of impact was designed to capture the time immediately following the impact with a series of 10-s exposures in the $\mathrm{R}$ filter. For the first hour of the night all filters were used, however starting around 5:00 UT images were only taken in R, and at 5:50 UT the exposure time was changed to $10 \mathrm{~s}$ from $20 \mathrm{~s}$. Images were taken at 5:52:08 UT and 5:52:39 UT with the hope of capturing the

\footnotetext{
4 The response of the SQIID detectors is non-linear for large counts. Thus, increasing the background increases the fraction of unrecorded incident counts. Due to the varying sky background at different wavelengths, the $\mathrm{H}$ and $\mathrm{K}$ bands were more severely undercounted than the $\mathrm{J}$ band during our observing run Using the equation for estimating the relative error given in the SQIID manual (http://www.noao.edu/kpno/sqiid/sqiidmanual.html), the J flux was typically undercounted by less than $0.2 \%$ while the $\mathrm{H}$ and $\mathrm{K}$ fluxes were undercounted by $2-2.5 \%$. However, the sky background was much higher on the night of impact than on any other night of the run, requiring corrections of $\sim 1 \%$ in J, 7-9\% in $\mathrm{H}$, and $4-5 \%$ in $\mathrm{K}$.
} 
Table 1

Observation summary

\begin{tabular}{|c|c|c|c|c|c|c|c|c|}
\hline UT date & $\begin{array}{l}\text { UT time } \\
\text { (h) }\end{array}$ & Location & Conditions & $\begin{array}{l}\text { Broadband } \\
\text { CCD filters }\end{array}$ & $\begin{array}{l}r \\
(\mathrm{AU})\end{array}$ & $\begin{array}{l}\Delta \\
(\mathrm{AU})\end{array}$ & $\begin{array}{l}\text { Comet airmass } \\
\text { range }\end{array}$ & $\begin{array}{l}\text { Standard star } \\
\text { airmass range }\end{array}$ \\
\hline July 2 & $5.1-5.3$ & $\mathrm{KPNO}^{\mathrm{a}}$ & Partly cloudy & JHK & 1.506 & 0.884 & $1.8-2.0$ & None \\
\hline July 2 & $5.5-7.1$ & OAN-SPM ${ }^{b}$ & Photometric & BVR & 1.506 & 0.884 & $1.9-4.2$ & $1.3-3.0$ \\
\hline July 3 & $3.8-6.8$ & KPNO & Photometric & JHK & 1.506 & 0.889 & $1.5-4.0$ & $1.4-1.7$ \\
\hline July 3 & $5.1-7.5$ & OAN-SPM & Not photometric & BVR & 1.506 & 0.889 & $1.7-4.1$ & $1.1-3.6$ \\
\hline July 4 & $4.1-7.3$ & KPNO & High sky background & JHK & 1.506 & 0.894 & $1.5-8.4$ & $1.4-2.0$ \\
\hline July 4 & $4.0-7.0$ & OAN-SPM & Photometric & BVR & 1.506 & 0.894 & $1.4-4.1$ & $1.2-4.0$ \\
\hline July 5 & $3.5-6.5$ & KPNO & Photometric & JHK & 1.506 & 0.899 & $1.4-3.5$ & $1.4-2.0$ \\
\hline July 5 & $4.6-7.0$ & OAN-SPM & Photometric & BVR & 1.506 & 0.899 & $1.4-4.3$ & $1.2-3.7$ \\
\hline July 6 & $3.7-6.5$ & KPNO & Photometric & JHK & 1.506 & 0.905 & $1.5-3.8$ & $1.4-2.1$ \\
\hline July 7 & $3.7-6.5$ & KPNO & Photometric & JHK & 1.506 & 0.910 & $1.5-4.0$ & $1.4-2.2$ \\
\hline July 8 & $3.7-6.5$ & KPNO & Photometric & JHK & 1.506 & 0.915 & $1.5-3.9$ & $1.4-2.3$ \\
\hline July 9 & $3.7-6.3$ & KPNO & Photometric & JHK & 1.507 & 0.921 & $1.5-3.7$ & $1.4-2.1$ \\
\hline
\end{tabular}

${ }^{\mathrm{a}} \mathrm{KPNO}=$ Kitt Peak National Observatory, Arizona (2.1-m).

b OAN-SPM = Observatorio Astronómico Nacional at the Sierra de San Pedro Mártir, Mexico (1.5-m).

impact (airmass $\sim 2.15$ ). A continuous set of 10-s exposures was taken starting $\sim 5: 53: 12 \mathrm{UT}$, with $26 \mathrm{~s}$ in between the start of consecutive exposures. At 6:06 UT we returned to 20-s exposures, and took these continuously until 7:03 UT when the comet was at 4.14 airmass.

The same standard star field was observed each night. The standard star field was observed immediately after Tempel 1 had set and was observed continuously until setting. The nightly range of airmasses for the standard star field and the comet are given in Table1. The PSF in B, V, R, and I typically evolved from $\sim 1$." 6 at low airmass to $\sim 2$." 5 at high airmass. A circular 3." 5 radius aperture was selected for all photometry to minimize the fraction of flux lost in the wings of the PSF.

The images were reduced using IRAF to subtract the bias and divide out the twilight flats for each night of observing. The standard stars were used to calculate atmospheric extinction corrections in each filter for each night (Landolt, 1992). Nightly median images were constructed by normalizing exposure times and taking the median value at each pixel.

\section{Lightcurve}

While no flash was discernable either in our guide cameras or in our images taken during impact, the comet brightened immediately after impact in all filters (R, J, H, and K) (Fig. 1). ${ }^{5}$ In

\footnotetext{
5 The two R band exposures immediately prior to impact appear brighter than any other pre-impact $\mathrm{R}$ band images. We explore several possibilities to explain this phenomenon: First, poor time synchronization could account for a 90-s lag. This seems unlikely since the times recorded in the image header match the times written by hand in the log sheets. Furthermore, the observatory clock was checked against several online time sources. Second, these two images were the first two taken on this night with 10-s exposures, raising the possibility that the difference in exposure time is responsible for the relative jump in brightness. A possible cause is uncertainty of the shutter speed, though the shutter travel time, estimated to be $10 \mathrm{~ms}$ (http://www.astrossp.unam.mx/resast/man $150 \mathrm{~cm} /$ manual-en.html), was very small compared to the exposure time. Since we returned to the 20-s exposures 14 min after impact, this would not affect our conclusions regarding the final increase in brightness (measured 25-35 min after impact). However, it would reduce the bluening of the $\mathrm{R}$ relative to the near-IR immediately af-
}

$\mathrm{R}$ it brightened at a nearly constant rate from about $1-20 \mathrm{~min}$ after impact, at which point it began to flatten out, reaching a plateau about 25 min after impact. In J, $\mathrm{H}$, and $\mathrm{K}$, it brightened slightly less steeply than $\mathrm{R}$ within 2 min after impact, brightened at a nearly constant rate from 2-14 min after impact, gradually flattened out from 14-25 min after impact, and reached a plateau around 25 min after impact. The lightcurve increased gradually and with increasing scatter during the remainder of our observations on the night of impact. Given the rising sky background, deteriorating seeing, and uncertain airmass correction at extremely large airmasses, this trend is not considered significant. For these reasons, we consider only those images taken at an airmass below 3.5 (approximately $35 \mathrm{~min}$ after impact) in all discussion of the lightcurve on the night of impact. Averaging the magnitude after it had leveled off but below 3.5 airmasses (from approximately $25-35 \mathrm{~min}$ after impact), the comet had brightened by 1.50 magnitudes in $\mathrm{R}, 1.35$ magnitudes in J, 1.33 magnitudes in $\mathrm{H}$, and 1.27 magnitudes in $\mathrm{K}$ for a 3." 5 radius aperture ( $\sim 2300 \mathrm{~km}$ at the comet). All of the ejecta were still within the 3." 5 radius aperture on the night of impact (discussed further in Section 6).

Fig. 2 shows the nightly median magnitude in all filters for each photometric night from July 3-9 UT, with one pre-impact and one post-impact median on July 4 UT in R, J, H, and K. Only images taken below an airmass of 2 were included, except for post impact observations on July 4 UT. Fluxes were corrected for the changing geometry $(r$ and $\Delta)$ from night to night. The lightcurve was not phased to the 40.7-h rotation period of the comet. Observations on subsequent nights revealed the comet slowly returning to normalcy after the steep increase in brightness following the impact on July 4 UT. By the night after impact, the comet was 0.37-0.39 magnitudes brighter in the visible and $0.24-0.26$ magnitudes brighter in the near-IR

ter impact by $\sim 0.08$ magnitudes. Third, the comet's non-sidereal motion was fast enough that a faint background star could have passed through the aperture during these two images, though no star was obvious. Finally, it is possible that they are simply two anomalous points preceding impact. Regardless, the first image taken after impact brightened substantially relative to these images, and no further anomalous behavior was seen. 


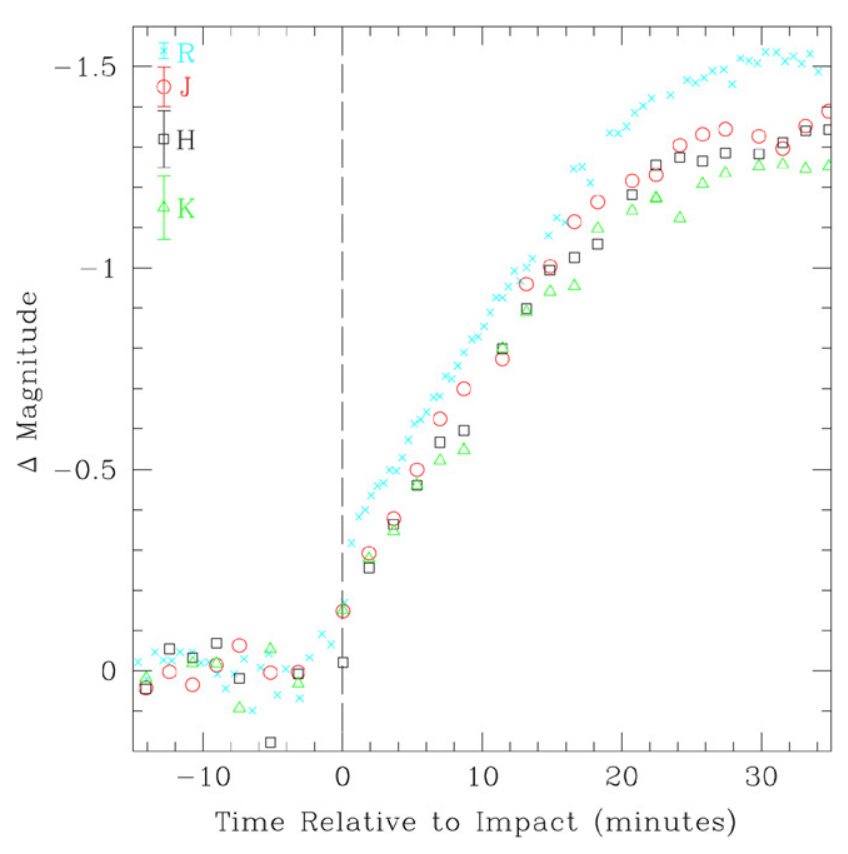

Fig. 1. Night of impact lightcurve for Comet Tempel 1 in a $3 . " 5$ radius aperture ( $\sim 2300 \mathrm{~km}$ at the comet). The pre-impact mean magnitude has been subtracted from each point. $\mathrm{R}$ band images are crosses, $\mathrm{J}$ band images are circles, $\mathrm{H}$ band images are squares, and $\mathrm{K}$ band images are triangles. The dashed vertical line represents the time of impact (5:52:02 UT). Typical 1- $\sigma$ error bars are shown in the legend.

(for a 3.' 5 radius aperture) relative to the pre-impact means on July 4 UT. By the fourth night after impact (July 8 UT) it had returned to nearly the pre-impact levels. The impact did not produce any substantial long term effects, and no new jets or active regions were created (Harker et al., 2005). On July 9 UT we observed the comet almost exactly three rotations after impact. Since the impact site was close to solar noon at impact, it is possible that the slight increase in brightness we observed on this night relative to July 8 UT was due to ice at the impact site responding to solar noon. However, this may simply be a normal night to night fluctuation in brightness.

\section{Color change}

The R-J, R-H, R-K, J-H, J-K, and $\mathrm{H}-\mathrm{K}$ color changes (in a 3." 5 radius aperture) on the night of impact for all $\mathrm{R}$-band images taken within $20 \mathrm{~s}$ of a JHK image are plotted in Fig. 3. While the errors for individual points are large, the bluening trend is evident in $\mathrm{R}$ relative to the near-IR in every image after impact. By 20 min after impact, the R-J, R-H, and R-K colors had bluened by 0.15 to 0.25 magnitudes, and begun to flatten. The near-IR color changes were less pronounced, but the bluening trend is evident. All but three post-impact $\mathrm{J}-\mathrm{H}$ points are blue, all but one post-impact $\mathrm{J}-\mathrm{K}$ points are blue, and all but four post-impact $\mathrm{H}-\mathrm{K}$ points are blue. Averaging the colors for all points after the lightcurve leveled off and below 3.5 airmasses yielded $\mathrm{J}-\mathrm{H}=-0.02$ magnitudes, $\mathrm{J}-\mathrm{K}=-0.08$ magnitudes, and $\mathrm{H}-\mathrm{K}=-0.06$ magnitudes.

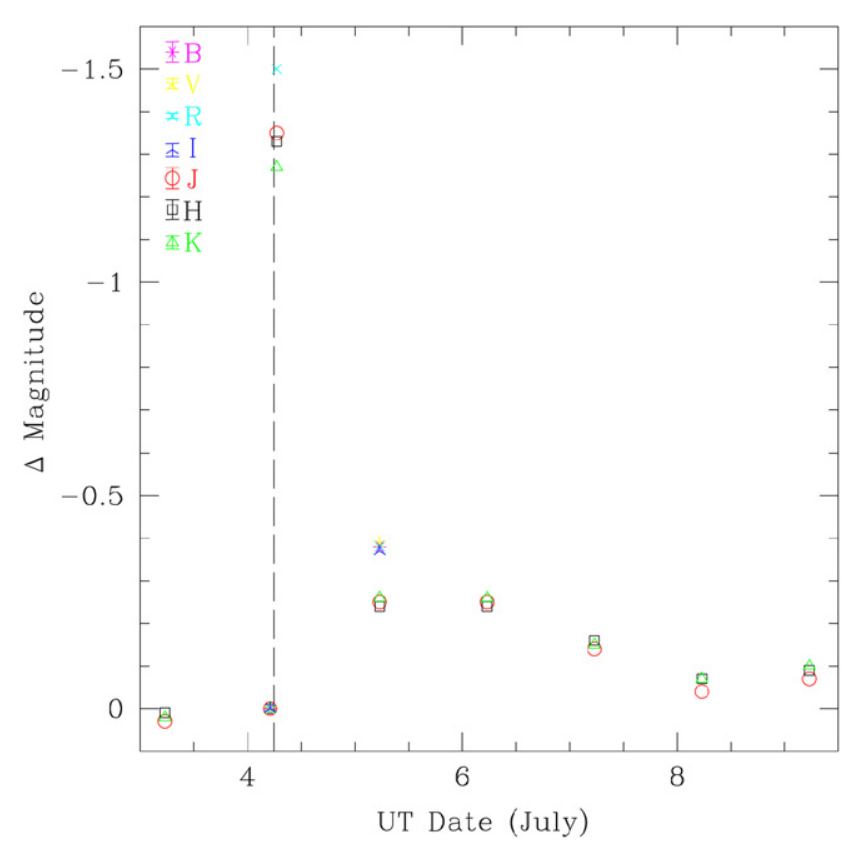

Fig. 2. Lightcurve for Comet Tempel 1 from July 3-9 UT in a 3." 5 radius aperture $(\sim 2300 \mathrm{~km}$ at the comet). Each point represents the median magnitude of all images below 2 airmasses, except for the night of impact where one pre-impact (all points prior to impact) and one post-impact (all images after the lightcurve leveled off and below 3.5 airmasses) median magnitude were calculated. All points had the pre-impact July 4 UT magnitude subtracted. B band images are six pointed stars, $\mathrm{V}$ band images are five pointed stars, $\mathrm{R}$ band images are crosses, I band images are three pointed stars, J band images are circles, $\mathrm{H}$ band images are squares, and $\mathrm{K}$ band images are triangles. $\mathrm{B}, \mathrm{V}$, and I images were only taken on July 4 UT prior to impact and on July 5 UT. The dashed vertical line represents the time of impact (5:52:02 UT). Typical 1- $\sigma$ error bars are shown in the legend.

Fig. 4 shows the long term color behavior after impact. The $\mathrm{J}-\mathrm{H}, \mathrm{J}-\mathrm{K}$, and $\mathrm{H}-\mathrm{K}$ colors for a $3 . ' 5$ radius aperture are plotted in the top three panels for the median of all images taken at an airmass less than 2 in a given filter on a given night. Separate pre- and post-impact median colors were calculated for July 4 UT (all images prior to impact and all images after the lightcurve leveled off and below 3.5 airmasses, respectively). Only images taken at an airmass less than 1.65 were included on July 7 UT, as the background jumped by $\sim 50 \%$ in all filters shortly thereafter.

As seen in the post-impact lightcurve (Fig. 1), the $\mathrm{J}-\mathrm{H}$, $\mathrm{J}-\mathrm{K}$, and $\mathrm{H}-\mathrm{K}$ colors bluened after impact, although the $\mathrm{J}-\mathrm{H}$ color change was roughly the same as the error bars. By the night after impact, the near-IR colors had returned to their preimpact levels. Fluctuations on subsequent nights were small compared to the post-impact increase in $\mathrm{J}-\mathrm{K}$ and $\mathrm{H}-\mathrm{K}$. Thus the only significant near-IR color change was the bluening immediately after impact and the return to "normal" color by the next night.

The bottom three panels of Fig. 4 show the R-J, R-H, and $\mathrm{R}-\mathrm{K}$ colors in a 3." 5 radius aperture on July 4 and 5 UT, the only nights for which we had photometric data from both KPNO and OAN-SPM. The R-J, R-H, and R-K colors bluened more dramatically than the near-IR colors immediately after impact. Unlike the near-IR color changes, the $\mathrm{R}-\mathrm{J}, \mathrm{R}-\mathrm{H}$, and $\mathrm{R}-\mathrm{K}$ col- 


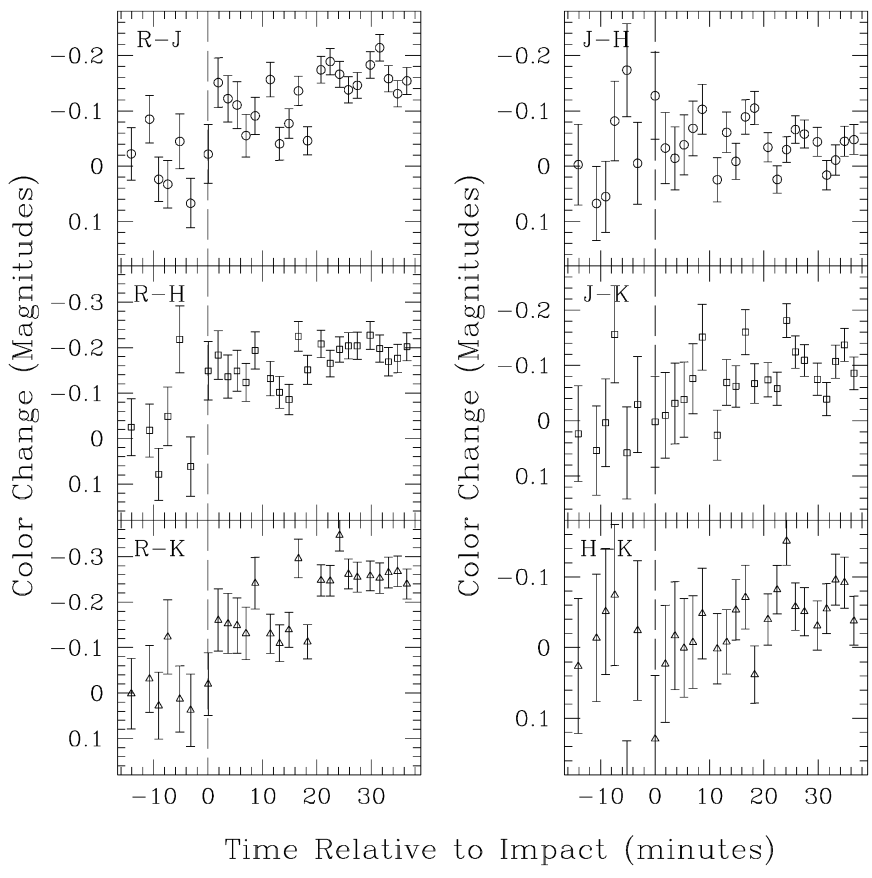

Fig. 3. Night of impact color plots for Comet Tempel 1 in a 3." 5 radius aperture ( $\sim 2300 \mathrm{~km}$ at the comet). The pre-impact mean color has been subtracted from each point. The left column is $\mathrm{R}-\mathrm{J}, \mathrm{R}-\mathrm{H}$, and $\mathrm{R}-\mathrm{K}$ from top to bottom, and the right column is $\mathrm{J}-\mathrm{H}, \mathrm{J}-\mathrm{K}$, and $\mathrm{H}-\mathrm{K}$ from top to bottom. Only times for which an R-band image was taken within $20 \mathrm{~s}$ of a JHK image are plotted. The dashed vertical line represents the time of impact (5:52:02 UT). 1- $\sigma$ error bars are plotted for each point.

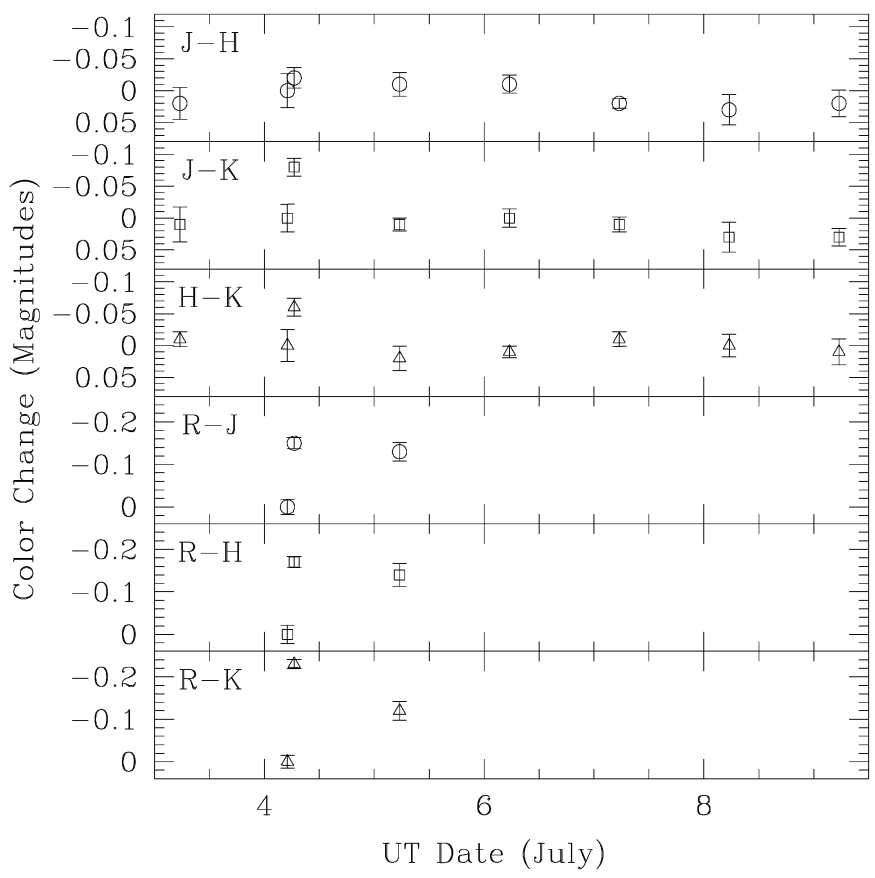

Fig. 4. J-H (top panel), J-K (second panel), H-K (third panel), R-J (fourth panel), R-H (fifth panel), and R-K (bottom panel) color computed for the run. Each point represents the median magnitude of all images below 2 airmasses, except for the night of impact where one pre-impact (all points prior to impact) and one post-impact (all images after the lightcurve leveled off and below 3.5 airmasses) median magnitude were calculated. The pre-impact median color has been subtracted from each point. A 3." 5 radius aperture $(\sim 2300 \mathrm{~km}$ at the comet) was used for all nights. 1- $\sigma$ error bars are plotted for each point. ors remained blue on the night after impact, though all three had reddened relative to their peak post-impact color.

\section{Coma morphology}

The ejecta were not spatially resolved in our images on the night of impact due to our low resolution and short observing window. By the night after impact, the ejecta were clearly visible to the southwest of the nucleus. Fig. 5 shows the nightly median image on July 5 UT in each of our filters with the pre-impact median and a $1 / \rho$ profile subtracted to enhance the ejecta relative to the ambient coma. A variety of processing techniques were used to enhance the ejecta, and this combination yielded the best image. We could not resolve the ejecta cone in our highest $\mathrm{S} / \mathrm{N}$ images ( $\mathrm{V}$ and $\mathrm{R}$, the middle two images of the top row), however the ejecta are nearly symmetric and show little evidence of being perturbed by solar radiation pressure. The ejecta are in the general southwest direction. The leading edge of the ejecta is seen at a projected distance of $18,000-20,000 \mathrm{~km}$.

Fig. 6 shows a sequence of nightly median $\mathrm{J}$ band images with the pre-impact median and a $1 / \rho$ profile subtracted. By the second night after impact (top right image), the ejecta are no longer symmetric, having been accelerated by solar radiation pressure towards the southeast (from an initial trajectory towards the southwest). The inner $\sim 10,000 \mathrm{~km}$ of the coma remains nearly as bright as the previous night, however the extended ejecta no longer have a well defined edge. By a projected distance of $\sim 25,000 \mathrm{~km}$ the ejecta become indistinguishable from the background. The reappearance of the nucleus in this image is an artifact of the stretch in the image display.

On subsequent nights (bottom row of Fig. 6), the ejecta are mostly in the southeast direction, the solar radiation pressure having deflected them from their original southwesterly direction. There is no obvious enhancement in the inner coma, and the ejecta are barely discernable above the ambient background noise. By July 8 UT (four nights after impact) the inner $\sim 15,000 \mathrm{~km}$ were no brighter than prior to impact, suggesting the ejecta had fallen back to the nucleus, moved beyond this region, or evaporated. The ejecta were still visible to the southeast, but were barely resolved above the background.

\section{Discussion}

The non-detection of the flash at impact was consistent with those of other ground-based observers (Meech et al., 2005b). Since a short (120 ms) flash was seen by the Deep Impact flyby spacecraft (A'Hearn et al., 2005b) and the impact site was visible from the Earth, it is likely that the contrast of the flash with the inner coma when integrated over the exposure time was not substantial enough to be visible by Earth-based telescopes.

The varying slopes of the lightcurve immediately after impact may correspond to the stages of excavation of the crater, the varying optical depths of the ejecta in the coma, and the rapid fragmentation and evaporation of the freshly excavated particles in the coma. We propose the following scenario to explain the observed lightcurve shape: The rapid brightening 


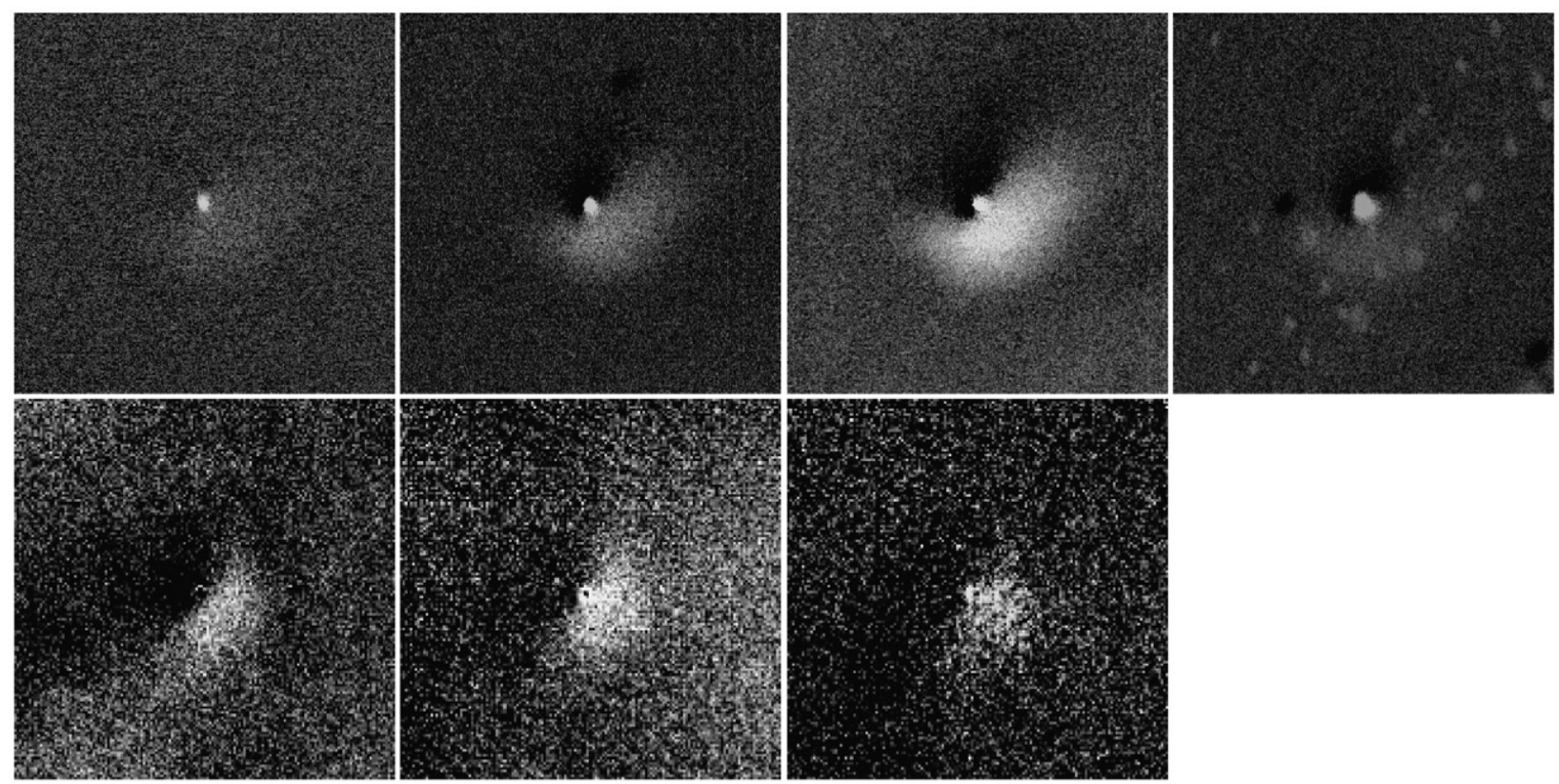

Fig. 5. Coma structure the night after impact. Median images from July $5 \mathrm{UT}$ in $\mathrm{B}, \mathrm{V}, \mathrm{R}$, and I (top row, left to right), and J, H, and K (bottom row, left to right) with the pre-impact median from July 4 UT and a $1 / \rho$ profile subtracted, stretched logarithmically. North is up and East is to the left. The Sun is towards the upper right at a position angle of $290^{\circ}$. The field is $\sim 68,000 \mathrm{~km}$ across each night. Background stars are seen moving across the I image.
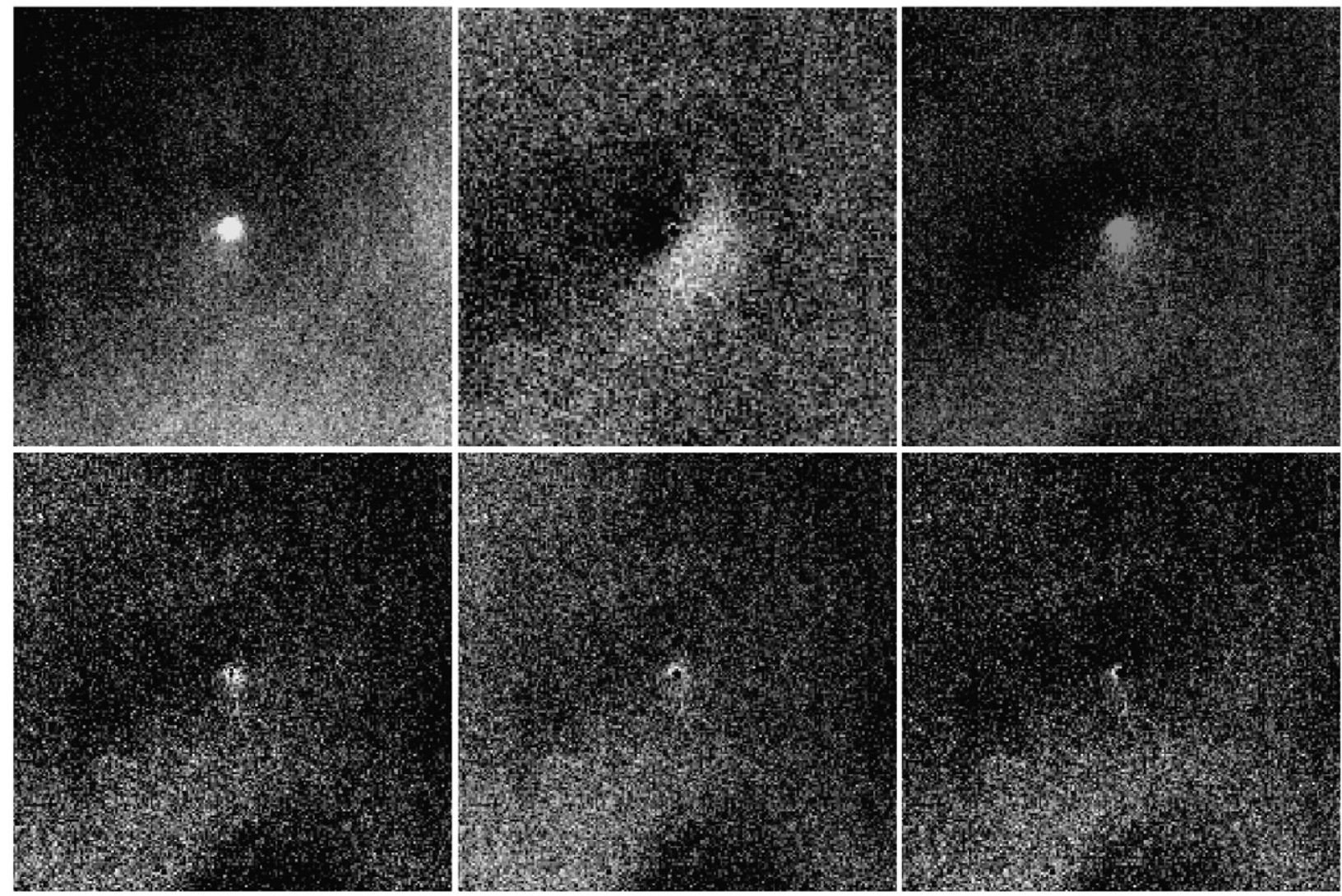

Fig. 6. Daily sequence of coma structure in the J band. Nightly post-impact median images from July 4-6 UT (top row, left to right) and July 7-9 UT (bottom row, left to right) with the pre-impact median from July 4 UT and a $1 / \rho$ profile subtracted, stretched logarithmically. North is up and East is to the left. The Sun is towards the upper right at a position angle of $290^{\circ}$. The field is $\sim 90,000 \mathrm{~km}$ across each night.

immediately after impact was likely due to the excavation of the crater by the impactor. New ejecta were likely thrown out for several min after impact during which time the lightcurve brightened slightly less rapidly than in the first post-impact im- 
age. The temporary flattening seen by Fernández et al. (2007) in $\mathrm{J}, \mathrm{H}$, and $\mathrm{K}$ 2-4 min after impact, was also evident in our data in a 1." 4 radius aperture, though the uncertainty is large. This may represent a transition point where the lightcurve went from a regime dominated by the excavation process to a regime dominated by the expansion of the ejecta and the fragmentation and evaporation of the particles. We hypothesize that during this time the ejection of new material slowed and the already ejected material was still optically thick. The steadily increasing brightness later than $\sim 4$ min after impact was due to the expansion of the ejecta, increasing the cross section for reflecting sunlight as the optical depth decreased. By about 25 min after impact, the ejecta had thinned enough that the maximum surface area had been achieved, and the lightcurve flattened out.

After the first $25 \mathrm{~min}$, the brightness was dominated by the effect of gravity on the ejecta and the lifetimes of freshly ejected ices. Particles ejected at velocities exceeding the escape velocity $\left(\sim 1.3 \mathrm{~m} \mathrm{~s}^{-1}\right.$ calculated from the derived mass and mean radius given by Richardson and Melosh, 2007) moved away from the nucleus, eventually passing beyond the apertures used for photometry. Particles ejected slower than the escape velocity fell back to the nucleus within a few days of impact. In situ (Sunshine et al., 2006) and Spitzer (Lisse et al., 2006) measurements soon after impact suggest the ejecta were predominantly $2-\mu \mathrm{m}$ particles. The timescale for ice to fragment or evaporate is short, of order a few hours for 2- $\mu \mathrm{m}$ grains of pure water ice in sunlight at 1.5 AU (Patashnick and Rupprecht, 1977). Impurities in the ice will make it darker, causing it to absorb more sunlight and greatly speeding up the sublimation process. Large grains will evaporate until they are a few $\mu \mathrm{m}$ in size (approximately the wavelength of the sunlight) and no longer absorb sunlight as efficiently, slowing the sublimation process down. Thus, the contribution of each particle to the observed surface area changed with time and was dependent on its ejection velocity, composition, and size.

These effects combined to cause the lightcurve to begin fading within the first hour after impact for observers with high spatial resolution and small apertures (see, e.g., Meech et al., 2005b for a longer timescale lightcurve), though our degraded seeing and extremely high airmass prevented us from observing it. The short lifetime of ices meant that by the night after impact, any brightness enhancement was due entirely to small refractory dust remaining in the coma. As the dust moved beyond our field of view, the coma gradually returned to its preimpact brightness, and the evidence of the impact disappeared. The leading edge of the ejecta, traveling at $\sim 0.23 \mathrm{~km} \mathrm{~s}^{-1}$ (discussed below) had already passed beyond the 3." 5 radius aperture on the night after impact, meaning the change in brightness was due entirely to particles ejected with a velocity less than $\sim 30 \mathrm{~m} \mathrm{~s}^{-1}$. By July $8 \mathrm{UT}$, the comet had returned to within 0.1 magnitudes of its pre-impact brightness in $\mathrm{J}, \mathrm{H}$, and $\mathrm{K}$ for a 3." 5 radius aperture (after correcting for the changing geometry) and did not fade further on July 9 UT, the last night of our run. Since particles ejected with an initial velocity of $\sim 7 \mathrm{~m} \mathrm{~s}^{-1}$ would have left the aperture by July $8 \mathrm{UT}$, any remaining enhancement was due to particles moving at roughly five times the escape velocity. As evidenced by the small increase in brightness rel- ative to the pre-impact level, these particles represented only a small fraction of the total ejecta. Alternatively, the remaining brightness enhancement may simply be normal brightness fluctuations in the comet, unrelated to the impact.

The bluening after impact can be explained by either an enhancement in small particles relative to the ambient coma or an increase in ice relative to refractory dust. Using $0.8-2.5 \mu \mathrm{m}$ spectra taken every $1.1 \mathrm{~s}$ from $12 \mathrm{~min}$ before impact to $14 \mathrm{~min}$ after impact, Fernández et al. (2007) observed the J-K color bluen in the first minute after impact, and become gradually bluer for the remainder of their observing window. We observed bluening immediately after impact in $\mathrm{R}$ relative to the near-IR. The J-H, J-K, and $\mathrm{H}-\mathrm{K}$ colors showed a bluening trend beginning after impact, though the error bars were as large as the effect until $\sim 14$ min after impact (Fig. 3). These results confirm that the ejecta were blue for at least $35 \mathrm{~min}$ after impact, and suggest that if the bluening was due to freshly excavated icy grains, the timescales for evaporation exceeded $35 \mathrm{~min}$.

To test if the bluening seen immediately after impact was attributable to ice, we estimated the color change expected for varying pathlengths of water ice. While scattering by grains will not be identical to absorption by bulk ice, a scattering calculation is beyond the scope of this paper, and this calculation is only intended as a plausibility argument. Water ice has large absorption bands from 1.4 to $1.7 \mu \mathrm{m}$ and 1.9 to $2.2 \mu \mathrm{m}$. The peak of the 1.4 to $1.7 \mu \mathrm{m}$ absorption feature falls between the $\mathrm{J}(1.131-1.402 \mu \mathrm{m})$ and $\mathrm{H}(1.535-1.809 \mu \mathrm{m})$ bandpasses (but closer to $\mathrm{H}$ ), however the peak of the second absorption feature falls within the $\mathrm{K}$ bandpass $(1.95-2.30 \mu \mathrm{m})$. As a result, ice absorption reduces the flux in the $\mathrm{K}$ band relative to $\mathrm{J}$ and $\mathrm{H}$ and in the $\mathrm{H}$ band relative to $\mathrm{J}$.

Grundy and Schmitt (1998) published transmission spectra from 1.0 to $2.7 \mu \mathrm{m}$ of water ice at temperatures between 20 and $270 \mathrm{~K}$. Using the spectrum at $270 \mathrm{~K}$, we calculated the expected $\mathrm{J}-\mathrm{H}, \mathrm{J}-\mathrm{K}$, and $\mathrm{H}-\mathrm{K}$ color changes for a variety of pathlengths. A pathlength of $18 \mu$ m yielded $\mathrm{J}-\mathrm{H}=-0.025$ magnitudes, $\mathrm{J}-\mathrm{K}$ $=-0.080$ magnitudes, and $\mathrm{H}-\mathrm{K}=-0.055$ magnitudes. These results are very similar to the observed color change on the night of impact: $\mathrm{J}-\mathrm{H}=-0.02$ magnitudes, $\mathrm{J}-\mathrm{K}=-0.08$ magnitudes, and $\mathrm{H}-\mathrm{K}=-0.06$ magnitudes. The surface temperature of the nucleus of Tempel 1 varied from $272 \pm 7$ to $336 \pm 7 \mathrm{~K}$ on the sunlit hemisphere (Groussin et al., 2007). While these temperatures are somewhat higher than the maximum temperature of the spectra published by Grundy and Schmitt (1998), the results did not change dramatically across the range of temperatures. Using the lowest published temperature, $20 \mathrm{~K}$, and the same pathlength yielded similar colors: $\mathrm{J}-\mathrm{H}=-0.032, \mathrm{~J}-\mathrm{K}$ $=-0.080$, and $\mathrm{H}-\mathrm{K}=-0.048$. Therefore we conclude that the results are reasonable, and that the color change seen after impact is consistent with water ice ejecta.

Alternatively, the bluening observed after impact may have been due to an increase in small particles rather than an increase in ice to dust. In this case, we would expect the color change to decrease gradually night by night as the fastest ejecta moved out of our field of view, leaving a smaller fraction of newly ejected particles relative to the ambient coma. $\mathrm{J}-\mathrm{H}, \mathrm{J}-\mathrm{K}$, and $\mathrm{H}-\mathrm{K}$ colors returned to their pre-impact levels by the night af- 
ter impact and remained that way the rest of the observing run (Fig. 4), while the R-J, R-H, and R-K colors remained blue on the night after impact, though they had reddened somewhat relative to their post-impact peaks. The persistence of color in the $\mathrm{R}$ relative to near-IR data suggests that the color change was due to some extent to the ejection of small particles.

As discussed above, the fastest moving ejecta would have moved beyond the 3." 5 radius aperture centered on the nucleus by the night after impact. Thus, the aperture used in Fig. 4 only observed the slowest moving components of the ejecta on the nights after impact. To measure the ejecta color on subsequent nights, it was necessary to use an aperture offset from the nucleus. To test whether the ejecta continued to be blue, we sampled the coma at a series of positions in the southwest quadrant (the initial direction of the ejecta) of our images out to a projected distance of $\sim 26,000 \mathrm{~km}$. We calculated the mean brightness at each position for all near-IR images with airmass less than 2 for each night of the run. A night by night lightcurve at each position was created, and the motion of the ejecta could be seen as the peak of the lightcurve appeared later at larger projected distances from the comet. While the passage of the ejecta through each field was evident, no color change was obvious in any of the apertures. Due to the rapid deterioration of the $\mathrm{S} / \mathrm{N}$ as the distance from the nucleus increased, we cannot rule out the possibility that the ejecta remained blue on subsequent nights. However, the lack of obvious bluening of the ejecta in the days after impact supports the idea that the bluening was short lived and attributable to freshly excavated ices.

While the comet brightened substantially in the hour immediately after impact, this increase was limited to the inner few pixels. No change in morphology was seen until the following night. Due to the deteriorating seeing, a projected velocity in excess of $0.45 \mathrm{~km} \mathrm{~s}^{-1}$ would have been required for the leading edge of the ejecta to appear beyond our PSF at the end of our observing on the night of impact. On the night after impact, the leading edge of the ejecta was measured by considering the excess counts in concentric annuli. It had reached a projected distance of $\sim 20,000 \mathrm{~km}$ in our first B, V, R, and I images taken on the night after impact, and at $\sim 18,000 \mathrm{~km}$ in our median $\mathrm{J}, \mathrm{H}$, and $\mathrm{K}$ images the night after impact. Thus, the fastest ejecta were moving at an average projected velocity of $0.20-0.23 \mathrm{~km} \mathrm{~s}^{-1}$ during the first day after impact. The fastest moving particles were seen in the visible images, which are sensitive to smaller particles than the near-IR. This is consistent with the findings by Harker et al. (2005) and Schleicher et al. (2006), however it may also be the result of the higher S/N in the visible images.

The $\mathrm{S} / \mathrm{N}$ was not sufficient to resolve the edge of the ejecta in the same manner by the second night after impact. However, a visual estimate of the edge of the ejecta in a median $\mathrm{J}$ band image suggests it had reached a projected distance of $\sim 25,000 \mathrm{~km}$ (though the distance varied with position angle). This implies the particles had decelerated to an average projected velocity of $\sim 0.08 \mathrm{~km} \mathrm{~s}^{-1}$ during the second $24 \mathrm{~h}$ after impact due to solar radiation pressure. There is visual evidence of solar radiation pressure by this time, and the motion is consistent with ejecta trajectories from the best model solution of Schleicher et al. (2006). On subsequent nights the leading edge of the ejecta was difficult to distinguish above the background, making it impossible to estimate velocities, though the ejecta were seen to be further accelerated by solar radiation pressure.

To look for changing structure on shorter timescales, we divided a median of all images taken in the first 30 min on July 5 UT by the median of all images taken during the last $30 \mathrm{~min}$ on the same night. Even in our highest $\mathrm{S} / \mathrm{N}$ images ( $\mathrm{V}$ and $\mathrm{R}$ ), this did not yield noticeable differences. Thus, while the morphology changed from night to night, it did not change rapidly enough to provide further insight into the ejecta expansion or the effects of solar radiation pressure on a given night.

\section{Conclusions}

As part of the worldwide observing campaign in support of the Deep Impact encounter, we obtained simultaneous J, H, and $\mathrm{K}$ band observations of the impact as well as high speed photometry in the R band. The comet brightened immediately after impact in all four bandpasses, brightening by $1.27-1.5$ magnitudes in a 3." 5 radius aperture. The lightcurve behavior was similar in all four filters, showing several changes in slope which may correspond to the excavation process and the evolution of the ejecta. The comet brightened in $\mathrm{R}$ relative to the near-IR, in $\mathrm{J}$ relative to $\mathrm{H}$ and $\mathrm{K}$, and in $\mathrm{H}$ relative to $\mathrm{K}$, indicating the ejecta were rich in water ice relative to refractory dust, overabundant in small grains relative to the pre-impact coma, or a combination of the two. The near-IR color changes $\sim 30 \mathrm{~min}$ after impact $(\mathrm{J}-\mathrm{H}=-0.02$ magnitudes, $\mathrm{J}-\mathrm{K}=-0.08$ magnitudes, and $\mathrm{H}-\mathrm{K}=-0.06$ magnitudes) were consistent with icy ejecta.

By the night after impact, the comet had faded from its peak post-impact brightness, though it remained 0.24-0.39 magnitudes brighter than prior to impact for a 3." 5 radius aperture. It remained blue in $\mathrm{R}$ relative to $\mathrm{J}, \mathrm{H}$, and $\mathrm{K}$, confirming that at least a portion of the color change was due to an increase in small grains. The $\mathrm{J}-\mathrm{H}, \mathrm{J}-\mathrm{K}$, and $\mathrm{H}-\mathrm{K}$ color changes seen in the first hour after impact had disappeared by the night after impact, and apertures offset from the nucleus to measure the ejecta in the days after impact saw no color change, though the $\mathrm{S} / \mathrm{N}$ was poor. This suggests that the ejecta contained short lived icy grains.

The leading edge of the ejecta was evident, moving at an average projected velocity over the first $24 \mathrm{~h}$ of $0.20-0.23 \mathrm{~km} \mathrm{~s}^{-1}$. Continued monitoring of the comet over the next four nights showed the effects of solar radiation pressure on the ejecta, as the ejecta were deflected from an initial southwesterly trajectory towards the southeast. By July $8 \mathrm{UT}$, Tempel 1 had returned to its pre-impact state with no obvious long term effects due to the impact.

\section{Acknowledgments}

We thank the referees, Jean Manfroid and Yan Fernández for their valuable suggestions, particularly regarding the handling of errors in high airmass data. We gratefully acknowledge support from the Kitt Peak National Observatory (NOAO) and the 
Observatorio Astronómico Nacional at the Sierra de San Pedro Mártir staff for helping during observations. We acknowledge the Horizons ephemeris service provided by JPL's Solar System Dynamics group. This publication makes use of data products from the Two Micron All Sky Survey, which is a joint project of the University of Massachusetts and the Infrared Processing and Analysis Center/California Institute of Technology, funded by the National Aeronautics and Space Administration and the National Science Foundation. This work was funded by NASA contract NASW00004 from NASA's Discovery Program.

\section{References}

A'Hearn, M.F., Belton, M.J.S., Delamere, A., Blume, W.H., 2005a. Deep Impact: A large-scale active experiment on a cometary nucleus. Space Sci. Rev. 117, 1-21.

A'Hearn, M.F., and 32 colleagues, 2005b. Deep Impact: Excavating Comet Tempel 1. Science 310, 258-264.

Fernández, Y.R., Lisse, C.M., Kelley, M.S., Dello Russo, N., Tokunaga, A.T., Woodward, C.E., Wooden, D.H., 2007. Near-infrared light curve of Comet 9P/Tempel 1 during Deep Impact. Icarus 187, 220-227.

Groussin, O., A'Hearn, M.F., Li, J.-Y., Thomas, P.C., Sunshine, J.M., Lisse, C.M., Meech, K.J., Farnham, T.L., Feaga, L.M., Delamere, W.A., 2007. Surface temperature of the nucleus of Comet 9P/Tempel 1. Icarus 187, 1625.

Grundy, W., Schmitt, B., 1998. The temperature-dependent near-infrared absorption spectrum of hexagonal $\mathrm{H}_{2} \mathrm{O}$ ice. J. Geophys. Res. 103, 2580925822 .
Harker, D.E., Woodward, C.E., Wooden, D.H., 2005. The dust grains from 9P/Tempel 1 before and after the encounter with Deep Impact. Science 310, 278-280.

Landolt, A.U., 1992. UBVRI photometric standard stars in the magnitude range 11.5-16.0 around the celestial equator. Astron. J. 104, 340-371, 436-491.

Lisse, C.M., and 16 colleagues, 2006. Spitzer spectral observations of the Deep Impact ejecta. Science 313, 635-640.

Meech, K.J., A'Hearn, M.F., Fernández, Y.R., Lisse, C.M., Weaver, H.A., Biver, N., Woodney, L.M., 2005a. The Deep Impact Earth-based campaign. Space Sci. Rev. 117, 297-334.

Meech, K.J., and 208 colleagues, 2005b. Deep Impact: Observations from a worldwide Earth-based campaign. Science 310, 265-269.

Patashnick, H., Rupprecht, G., 1977. The lifetime of ice particles in the Solar System. Icarus 30, 402-412.

Persson, S.E., Murphy, D.C., Krzeminski, W., Roth, M., Rieke, M.J., 1998. A new system of faint near-infrared standard stars. Astron. J. 116, 2475 2488.

Richardson, J., Melosh, H.J., 2007. Determining Tempel 1's gravity, mass, and density via the ballistic behavior of the Deep Impact solid ejecta plume. Icarus. In press.

Schleicher, D.G., Barnes, K.L., Baugh, N.F., 2006. Photometry and imaging results for Comet 9P/Tempel 1 and Deep Impact: Gas production rates, postimpact light curves, and ejecta plume morphology. Astron. J. 131, 11301137.

Skrutskie, M.F., and 30 colleagues, 2006. The Two Micron All Sky Survey (2MASS). Astron. J. 131, 1163-1183.

Sunshine, J.M., A'Hearn, M.F., Groussin, O., Feaga, L.M., Li, J.-Y., Schultz, P.H., and Deep Impact Science Team, 2006. Water ice on Tempel 1: Before, during, and after the impact event. Lunar Planet. Sci. 37. Abstract 1890.

Yeomans, D.K., Giorgini, J.D., Chesley, S.R., 2005. The history and dynamics of Comet 9P/Tempel 1. Space Sci. Rev. 117, 123-135. 